solvent as the mobile, phase ${ }^{6, ?}$. Zaffaroni has observed that the rate of running of the steroids is closely related to the number and nature of groups containing oxygen in the molecule. We have found that the hydrophilic nature of the molecule can be approximately described by the expression

$$
z=2 \cdot 5 x_{3}+1 \cdot 5 x_{17}+x_{11},
$$

where $x=1$ for $=\mathrm{O}$ and $3 / 2$ for $\mathrm{OH}$ groupings, the subscript number that describes $x$ referring to the position of the substitution of these groupings. This expression holds for compounds possessing typical cortical steroid substitutions, including the $\alpha$-ketol grouping at the 20,21 position. The rate of running of the steroids is given by a function of $z$, the particular form of which is determined by the actual solvents used. However, for any system, the comparison of the values of $z$ between different steroids gives their sequence ; for example, Reichstein's $S$ ( $\Delta^{4}$ pregnane $17,21$ diol 3,20 dione, $z=4 \cdot 75)$, Kendall's $E\left(\Delta^{4}\right.$ pregnane 17,21 diol $3,11,20$ trione, $z=5 \cdot 75$ ), and Kendall's $F\left(\Delta^{4}\right.$ pregnane $11,17,21$ triol 3,20 dione, $z=6.25$ ) move in the sequence of their $z$ values, $F$ moving the slowest. The active mineralocorticoid runs slightly faster than Kendall's compound $E$ and much slower than Reichstein's compound $S$; it must therefore have a $z$ value between 5.75 and $4 \cdot 75$. These requirements are not fulfilled by substitutions at the 11 and 17 positions only, and together with the ultra-violet spectroscopic evidence indicate the presence of an $\mathrm{OH}$ group in the 3 position. This is further indicated by the active compound forming a di- or poly-acetate on acetylation.

of the twenty-eight steroids isolated from the adrenal cortex only two, Reichstein's compound $P$ (allopregnane $3,17,21$ triol 20 -one, $z=6 \cdot 00$ ), and $R$ (allopregnane $3,11,21$ triol 20 -one, $z=5 \cdot 25$ ) nearly satisfy the required $z$ values, have an $\alpha$-ketol grouping at the 20,21 position and yet are not 3-keto compounds. Zaffaroni has observed that Reichstein's $P$ moves slightly more slowly than Kendall's $E$. We have confirmed this in adrenal extract using the detection method of R. Neher and A. Wettstein ${ }^{8}$ for 11-deoxy compounds. It would seem, therefore, that, of the known cortical steroids, only Reichstein's compound $R$ (allopregnane $3 \beta, 11,21$ triol 20 -one) could be the active compound. There is some evidence that epimers of the same compound run at slightly different rates ; for example, Reichstein's $C$ (allopregnane $3 \alpha, 11,17,21$ tetrol 20 -one) runs slightly faster than Reichstein's $V$ (allopregnane $3 \beta, 11,17,21$ tetrol 20-one). There is, therefore, a further possibility that the active compound is an epimer of Reichstein's $R$ or $P$.

This further investigation has fully confirmed the earlier, more indirect work ${ }^{1,2}$, which showed that the mineral activity of beef adrenal extract was not due. to any of the known biologically active cortical steroids. Positive characterization of the structure of the compound must await the isolation of larger amounts of material; but it seems certain that this compound does not possess the $3 \alpha, \beta$ unsaturated ketonic grouping which has previously been assumed to be essential for high activity in mineral metabolism.

We wish to thank Allen and Hanburys for supplies of their adrenal extract 'Eucortone' and Prof. 'T. Reichstein for gifts of steroids. We have had the advantage of many discussions with Drs. Kellie and Jepson of this Medical School, and we are also grateful to Profs. E. C. Dodds and J, E, Roberts for their constant interest and advice. P. Avivi, S. Graves and M. Woodford gave us invaluable experimental assistance.

HILARy M. GRUNDY

Courtauld Institute of Biochemistry,

J. F. TAIT

Department of Physics Applied to Medicine, Middlesex Hospital Medical School, London, W.1. Feb. 21.

${ }^{1}$ Grundy, H. M., Simpson, S. A., and 'Tait, J. K., Lancet, 262, 122 (1952).

${ }^{2}$ Simpson, S. A. and 'Lait, J. F., Endocrin. (in the press).

${ }^{3}$ Zaffaroni, A., Burton, R. B., and Keutmann, E. H., Science, 111, 6 $(1950)$.

"Morton, R. A., "The Application of Absorption Spectra", 47 (London. 1942).

$\checkmark$ Burton, R. B., Zaffaroni, A., and Keutmann, E. H., J. Biol. Chem., 188, 763 (i951).

- Bush, I. E., Biochem. J., 50, 370 (1952).

7 Zaffaroni, A., and Burton, R. B., J. Biol. Chem., 193, 749 (1951).

${ }^{8}$ Neher, R., and Wettstein, A., Helv. Chim. Acta, 34, 2278 (1951).

\section{Separation of Urinary Estrogens by Micro-Partition Chromatography}

IN a recent report elsewhere ${ }^{1}$ we have shown that the oestrone and cestradiol in the phenolic fraction of urinary extracts can be separated satisfactorily, in microgram quantities, by means of a silica column, using aqueous sodium hydroxide as the stationary phase and benzene as the moving phase. We were at that time unable to deal with the ostriol fraction chromatographically, and separated it chemically by the method of Bachman and Petit ${ }^{2}$; this, however, was a tedious process and left unsolved the problem of purification so essential for satisfactory determination by fluorimetry using phosphoric acid. We have now succeeded in overcoming both these difficulties in one operation, and can separate the three fractions from the crude phenolic extract, achieving a satisfactory degree of purification in the process, on a single silica column.

As before, the column consists of $1.25 \mathrm{gm}$. of 'Celite 535' (Johns Manville Co., Ltd.), previously washed with a mixture of chloroform and butanol $(3: 1)$. The stationary phase is $2 \cdot 3 N$ sodium hydroxide $(1 \mathrm{ml}$.$) . Equilibrated benzene is run through under$ pressure to separate the cestrone and oestradiol as previously described. When the last of the moving phase has reached the top of the 'Celite' column, the pressure is let down and the vessel originally containing the phenolic extract is washed with four lots of $0.5 \mathrm{ml}$. of chloroform-butanol mixture $(3: 1)$, previously equilibrated with $2 \cdot 3 \mathrm{~N}$ sodium hydroxide, so as to take up any œstriol which may not have dissolved in the benzene. The pooled chloroformbutanol washings are then added to the top of the column and forced through, followed by a further $2 \mathrm{ml}$. of chloroform-butanol mixture to wash down the sides of the tube holding the column. Finally, the rest of the new moving phase (originally $55 \mathrm{ml}$.) is passed through the column. Our fraction cutter produces fractions of $4.2 \mathrm{ml}$., and we find the cestriol to be in fractions $3-7$ of the 'second run'.

Further details of the method, and the results obtained with it, will be published elsewhere.

MARgareT I. STERN

G. I. M. SWYER

University College Hospital Medical School, London, W.C.1. Dec. 11.

1 Swyer, G. I. M., and Braunsberg, H., J. Endocrinol., 7, 1x (1951). 2 Bachman, C., and Petit, D. S., J. Biol. Chem., 138, 689 (1941). 